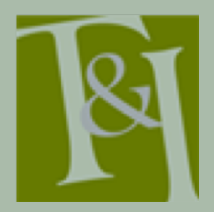

The International Journal for Translation \& Interpreting Research trans-int.org

\title{
The online computer-assisted translation classroom
}

\author{
Viktorija Bilić \\ University of Wisconsin-Milwaukee, USA \\ bilic@uwm.edu
}

DOI : 10.12807/ti.112201.2020.a08

\begin{abstract}
In the modern translation industry, all actors involved in the translation process communicate and collaborate increasingly - and oftentimes entirely - online using modern computer-assisted translation and project management software solutions. New translation technology needs new approaches to translator education and the Online Computer-Assisted Translation Classroom was designed to address this gap. Students are taught how to use the latest translation technology through hands-on remote software use and collaborative translation exercises. This article looks at the pedagogy of online translation teaching. It specifically discusses the teaching of translation technology and terminology management, drawing on process-oriented approaches to translator training and the method of cooperative translation.
\end{abstract}

Keywords: online teaching; translator training; translator competence; computerassisted translation; terminology management

\section{Introduction}

Today, many translators, especially in the U.S., work remotely as freelancers (McKay, 2006), communicating with agencies and clients entirely online. Virtual networking and creating a strong and professional online presence are essential in the translation industry, especially for freelance translators (McCallum \& Puchner, 2017). It is recommended that both new and established translators present themselves as professionals on their own websites and actively communicate with colleagues and potential clients on translator networking sites such as LinkedIn and ProZ (McCallum \& Puchner, 2017). Similarly, communicating actively with their instructor and fellow classmates is important for students in the virtual classroom as well. In addition to excellent communication skills, professional competence in the use of computer-assisted translation (CAT) tools is required for most translation jobs. Since translation technology is constantly evolving (Bowker, 2014, p. 88), it is crucial to be aware of recent developments in the field. Current trends in translation technology, for example, include post-editing machine translation output, project management integration in CAT software, as well as crowd translation and cloud-based CAT tool use (Chan, 2018). Translator training at the university level needs to reflect current technological developments to best prepare students and future translators for the demands of working in today's fast-paced translation industry (Kornacki, 2018). New approaches to translation technology require innovative approaches to translator education and thus the Online Computer-Assisted Translation Classroom was designed to teach students how to use the latest translation technology through hands-on remote software use while learning from their instructor and collaborating with their classmates in the virtual classroom (Bilić, 2015; Bilić, 2017). Research has been conducted on the use 
of CAT tools in both educational and professional settings (Keller, 2014; Krüger, 2016; Starlander \& Morado Vázquez, 2013). Research has also explored online learning versus blended learning or online learning versus faceto-face teaching (Larson \& Sung, 2009; Lim, Morris, \& Kupritz, 2007). It is beyond the scope of this article to compare teaching modes. Instead, this article focuses on teaching CAT tools all online in an all online Graduate Program in Translation at the University of Wisconsin-Milwaukee. There is little research available on teaching Computer-Assisted Translation in a fully online environment. This paper attempts to fill this gap by evaluating teaching methods that have proven successful over a period of four years. The success rate is measured based on the course objectives, which include gaining hands-on translation practice using SDL Trados Studio $^{1}$ and $\mathrm{memoQ}^{2}$; learning translation and terminology software, and troubleshooting in an online environment; and passing the Level 1 SDL Trados Translator Certification exam. This article will first outline the advantages and challenges of teaching translation technology in the virtual classroom. It will then focus on strategies for creating and maintaining an interactive online classroom, namely by integrating real-life working scenarios that enable students to use the latest CAT software on a course server and to collaborate on translation projects entirely online. This hands-on strategy for teaching CAT software is based on processoriented approaches to translator training (Kußmaul, 1995) and the method of cooperative translation (Kornelius, Orbán, \& Stewart, 2010). The quality of the end-product is assured by integrating detailed modules, including text analysis and research, terminology management, editing in translation, as well as timely feedback from the course instructor. This contribution puts special emphasis on teaching terminology management to graduate students of Translation in the online environment.

\section{Teaching translation technology online: Advantages, challenges, and strategies}

The online computer-assisted translation class has been developed as an entirely online graduate course in computer-assisted translation for distance students working in several different language pairs (Bilić, 2016-2019). There are advantages to teaching translation in the virtual classroom. Since they are required to market themselves as professional translators online, students and aspiring translators are actively creating an online presence in the online classroom. Sun notes that fully online learning comes with its own set of challenges, because, as opposed to blended and hybrid learning models, difficulties cannot be solved by reverting to traditional classroom teaching methods (Sun, 2014, p. 20). Online learning is very flexible and attractive to students who work and study, while at the same time online learning becomes much more a function of the learner compared to the traditional on-site classroom setting (Terando, 2014). The fully online learning experience is increasingly learner-centred. Instructors put a major focus on course design and constant (re-)configuration of the students' fully online learning environment in technology-enhanced virtual classroom settings (Sun, 2016, pp. 349-350). Students must actively engage with the course content and instructors need to facilitate student participation by integrating discussion fora, group projects,

\footnotetext{
${ }^{1}$ SDL Trados Studio is a computer-assisted translation tool by the British company SDL. The acronym SDL stands for Software and Documentation Localization. Trados stands for TRAnslation \& DOcumentation Software. SDL Trados Studio includes the terminology management software SDL MultiTerm.

${ }^{2}$ MemoQ is a computer-assisted translation tool by the Hungarian Kilgray company, founded by Balázs Kis, István Lengyel and Gábor Ugray, three language technologists with experience in the translation industry.
} 
and synchronous (live) sessions (Leone, 2015; Lowe, 2015). Gracia García notes:

With e-learning, the teachers must communicate with each student individually, and students need to participate in every activity in order to earn credit. In addition, in a truly interactive online course, students are motivated differently, because their audience is not the teacher alone, but rather their fellow students. Distance can bring teachers and students closer. (2005, pp. 446-447).

According to Susanne Göpferich and Riitta Jääskeläinen, the application of computer-assisted translation software is one of the three main translator competencies (Göpferich \& Jääskeläinen, 2009, p. 17). Therefore, courses on computer-assisted translation are an invaluable part of any translator education program. Learning translation technology in an online setting, as opposed to a traditional classroom setting, mimics most translators' real-life working situations and prepares students for their careers in the translation industry. However, teaching translation online holds various challenges. Developing and teaching a graduate online course in Translation is more time-consuming than developing and teaching the same course content as a face-to-face class (Lowe, 2015; Terando, 2014). All course material must be more thorough and complete than it would have to be on-site for example, and it is crucial to create and maintain an interactive online learning community (Gracia García, 2005; Terando, 2014). In the online CAT classroom, there is the added challenge of students accessing translation technology software remotely. However, it is highly beneficial for students to access the software on a university server instead of working with site licenses for their home computers. The use of a server enables the instructor to connect the workstations to one another, so that students can collaborate on projects in real-time using server versions of CAT tools (e.g. memoQ server). Students access the CAT software on a dedicated virtual online lab via a remote desktop protocol connection (RDP). ${ }^{3}$ This also means that the instructor and the students solve technological problems entirely online using modern technology like Skype, project management functions of CAT tools, online rooms on the course platform (e.g. Blackboard Collaborate), email, and a separate online discussion board on the course platform dedicated to troubleshooting CAT software issues. Another strategy for successful online CAT class delivery whose goal is building an online community includes a strong instructor presence through virtual office hours and on-site office hours, adequate and timely instructor feedback, and effective use of online discussion fora. In addition, similar to traditional classroom settings, it is crucial to set and adhere to course schedules and deadlines and to facilitate collaborative translation and group work among students in the online environment as well. In order to help students to recognize the benefits of CAT technology, we work with specialized and repetitive texts that lend themselves to CAT use and require consistent terminology. However, the course also puts a focus on potential disadvantages of CAT tools by discussing these in the fora as well. The hands-on approach used to teaching CAT software is based on processoriented approaches to translator training (Kußmaul, 1995) and the method of cooperative translation (Kornelius et al., 2010). There are several methods to conduct translation process research and gain insights into the "black box" of translation, including think aloud and dialogue protocols, questionnaires and interviews, video and screen recording or eye-tracking (Göpferich \& Jääskeläinen, 2009). For the purposes of evaluating the effectiveness of

\footnotetext{
${ }^{3}$ The remote desktop protocol (RDP) connection enables students to connect to the class server from their personal computers (PC or MAC) that are connected to the Internet. The class server (virtual translation lab) is a Windows environment that hosts the CAT software used in the course. Students each have their own virtual workstation, running SDL Trados Studio, SDL MultiTerm, and memoQ. Students then work on the virtual workstation in the same way they would utilize any PC.
} 
teaching computer-assisted translation in the virtual classroom, student course evaluation questionnaires, real-time screensharing, and data gathered from remote access to students' CAT class workstations have been evaluated. In the CAT class online setting, my goal is to foster a collaborative environment by creating a student-centred online community. Students learn about terminology management and theoretical aspects of the translation workflow and develop CAT tool competencies by working in (online) settings that mirror professional translation practice. The course is constantly being updated to ensure that students have an optimal course experience. Since we work with the latest versions of each CAT tool, all lectures, homework assignments, quizzes and screencasts are updated to reflect any changes in new software versions. Students work with the latest versions of industry-leading CAT technology and become familiar with the use of CAT tools and terminology management tools in the translation lifecycle from document receipt and preparation to final delivery. Using server-based editions of memoQ, student translation teams collaborate entirely online and gain industry-relevant experience in the roles of project managers, translators, and editors.

\section{The online computer-assisted translation class}

\subsection{Course design and course goals}

Course design is the key to effective online learning and student engagement (Lowe, 2015; Parker, 2013; Terando, 2014). Online course platforms that are used for online course delivery, such as Desire2Learn and Canvas, allow instructors to conduct courses through (narrated) lectures, readings, screencast tutorials, class discussion fora, hands-on practice homework assignments, and quizzes. At the core of every online course is a well-organized online learning platform with a detailed syllabus and clear and complete course materials. A core element of any class is a clearly structured course syllabus. Compared with classes that are designed for on-site classroom delivery, syllabi for online classes need to be more detailed. Syllabi for online graduate-level translation classes must include a detailed description of the class requirements, schedules, due dates and deadlines for homework assignments (Lowe, 2015; Terando, 2014). Due to the constantly developing translation technology software, onsite and online CAT classes must be updated each year to reflect changes in the newest software versions and other new trends in translation technology. The online CAT course consists of 15 weekly modules, including lectures, readings, screencast tutorials, homework and final project assignments, a discussion forum component, and quizzes. While the online nature of the course allows students to complete activities at their own pace, time management is crucial as students can expect to spend about 9-12 hours a week on the course (depending on their comfort level with translation technology software). In the virtual CAT classroom, each course module spans one week focusing on one specific topic, from an introduction to CAT technology at the beginning of the semester to server-based translator/editor projects toward the end of the term. The weekly topic is also considered in the discussion forum component of the class, where students compose answers to questions posed by the instructor or other students and engage in critical discussions. There are clear guidelines for discussion posts on what constitutes a critical reflection. These are outlined on the course syllabus. Comments like "good point" etc. are not counted as critical reflection posts. Perhaps most importantly, engaging in the discussion forum is a way for students to reflect on their learning (Leone, 2015). The instructor answers questions directly in the forum and provides a wrap-up document at the end of each week, summarizing the findings and lessons learned from each module. Students benefit by learning from each other and helping fellow students by providing tips on how to tackle software errors etc. Teaching translation should 
always include plenty of practice translation assignments. The same is true for teaching translation technology. The weekly hands-on CAT software homework assignments have been considered the most important element of the online CAT class by all graduate translation students who have taken the course evaluation survey. Over the course of four semesters (2016-2019), 57 Translation graduate students have taken the online CAT class. All of the survey respondents (46 of the 57 graduate students) remarked that the practical CAT software exercises were among the most beneficial course components (UWM, 2019). Each week, students work on translations and terminology databases using CAT software and learn how to effectively use CAT tools through handson successive tasks. The instructor provides detailed personal feedback on these assignments on a weekly basis to make sure that students are using CAT software correctly. For the instructor, this often includes troubleshooting CATrelated software user errors, rebooting the remote workstation that students are accessing via remote desktop protocol (RDP) or troubleshooting errors with the students using Skype screen sharing or exchanging screenshots that illustrate the software error students are experiencing. Since the class is taught entirely online, the instructor and the students discuss and solve any CAT technologyrelated question and system errors in a dedicated troubleshooting forum on the online course platform or via real-time Skype screen sharing. Problems that frequently occur include user errors, such as misplacing files in SDL Trados Studio's file/folder structure or system errors, such as temporary bugs that can occur after system updates that cause the software to stop functioning correctly. Students often post screenshots of software error messages and CAT-related questions in the asynchronous discussion board that are then answered by the instructor or other students in a timely manner. Alternatively, students may contact the instructor during Skype office hours, when problems are solved via real-time Skype screen sharing. The instructor then guides students through the troubleshooting process by accessing the student's workstation, checking the system for accurate functionality, and going over solutions to software troubles with students online and in real time. This way, students learn CAT technology troubleshooting skills that are highly beneficial as they prepare to enter the translation industry as freelance translators who often collaborate with clients and translation agencies entirely online. Finally, the online setting lends itself to incorporating group work to foster a collaborative environment and an interactive online teaching and learning experience. Online teachers must take full advantage of the online course platform and all its integrated components (lectures, readings, video screencasts, tutorials, homework exercises), as well as online meeting rooms (Blackboard Collaborate) to foster active student participation. Instructor feedback is highly important in the online classroom and especially when teaching the use of complex software programs. Students usually receive feedback within one or two days of submitting homework exercises or quizzes. The discussion forum also creates a space where students can ask questions, clarify doubts, troubleshoot software issues, and share interpretations of assigned readings. Discussions in the online forum, online meetings using tools such as Blackboard Collaborate, as well as Skype office hours with screen sharing, provide an opportunity for students to interact with the instructor and classmates. The course is designed to teach future translators the main features of translating and editing using the latest versions of SDL Trados Studio and memoQ. This includes the use of translation memory (TM) and alignment features, as well as terminology database (TB) creation and effective terminology management using market-leading CAT tools. Students also obtain certifications for SDL and memoQ as part of the course which is beneficial as they enter the translation market. Each week, we focus on a different CAT tool topic that relates not only to practical aspects of using the software but also to areas that are crucial in the translation industry, including translation rates, translation memory ownership and copyright, and the 
compatibility of different CAT systems. In addition to gaining hands-on translation practice using the newest versions of SDL Trados Studio, SDL Multiterm, and memoQ, students learn the important skill of troubleshooting technology issues in an online environment. Throughout the semester, students work on weekly CAT tool exercises and they work on a final translation project of their choice. This way, they build a termbase and a translation memory in a specialization they are interested in, such as medical, legal or technical translation. At the end of the class, small groups of students investigate and present on a translation technology of their choice (e.g. a CAT tool not discussed in class, a project management (PM) software etc.). Throughout the course students also take quizzes which prepare them for taking the SDL and memoQ certifications. In all, students enhance their knowledge of CAT tools by working on both weekly and semester-long exercises and by collaborating and communicating online with their instructor and their classmates via the course platform.

\subsection{Teaching the use of CAT technology: Terminology management as an example}

Computer-assisted translation technology is complex and, in many cases, intimidating for students new to CAT software. Thus, CAT technology is best taught using a step-by-step approach with exercises first focusing on the basic features and then on more elaborate software functions (scaffolding). The goal is to equip students with the step-by-step instructions and screencast tutorials needed to complete exercises. Students gain further software experience by working on a larger text of their choice throughout the semester. They first prepare and format this source text for CAT tool use, and they then build a termbase and translate the source text into their target language. Finally, students write two essays reflecting on the advantages and disadvantages of using CAT software for producing their target texts. As Mellinger notes, "translation students must be aware of the context in which translation memory tools are used and the impact that their use has on the translation itself." (Mellinger, 2014, p. 112). The reflection instructions were created with that goal in mind, and ask students to observe the advantages and disadvantages of terminology management and translation memory use. In their reflection essays, students pointed out that there were benefits to working with a side-by-side editor in a CAT program and that formatting is automatically carried over from the ST to the TT segments. They noted that using termbases helped to keep terminology consistent while translating repetitive and specialized source texts. Generally, the termbase function was viewed very favourably. Students were also encouraged to outline potential downsides of CAT tool use as these were also discussed in class. Here, many students mentioned that there is a risk of translators automatically accepting fuzzy matches and full matches that are suggested by the CAT tool's TM, even if the matches don't fit the context. The segmentation removes individual sentences from their contexts, which has often been noted as a disadvantage by students in the class. Students also wrote about technology issues they occasionally encountered, such as software freezes or crashes. Quality management in translation is discussed in the course as part of various steps in the translation lifecycle, from file preparation, research, and terminology management to proofreading and editing. In the fora, students discuss how to address these potential downsides of CAT tool use, for example by using the preview function to see target segments in their full context. Through practice exercises, in which students are paired up as teams of translators and editors working in the same language pairs, students gain practice applying quality management guidelines to the computer-assisted translation process. One important step to assuring translation quality in specialized translation is careful terminology management. 
In the following, I will describe my step-by-step approach to teaching CAT technology using the module on terminology management in week 5 as an example. The module consists of a lecture on terminology, readings, homework assignments, and a discussion component focusing on the importance of terminology in specialized translation. In addition, students post questions each week in the separate optional forum dedicated to solving and troubleshooting software issues. The hands-on practice exercise for week 5 focuses on creating an SDL MultiTerm termbase for translating the final project source text. Naturally, as a means of quality management, terminology work needs to be done before any attempt at translating a text. Proper terminology-management is time-consuming. Yet, terminology work gains increasing significance in (specialized) translation. Many international institutions and large corporations employ specialized terminologists to achieve high-quality terminology management. Standardized and well-maintained terminology is a key to successful communication, internationalization, and translation (Childress, 2007, p. 43). Through the readings, the lecture, and most importantly the homework assignment for the week, students learn and experience that terminology management is worth the effort and why it plays such an important role in the translation industry. Terminology management enables all parties involved in the translation lifecycle to use terminology correctly and consistently. Sauberer (Director of the International Network for Terminology) estimates that about 40 percent of translation time is terminology research: Documented terminology reduces time spent on re-research (Sauberer, 2009). In addition, terminological changes in large translation projects can be very costly. Clarifying terminology before working on a project reduces query time later on (Childress, 2007). Above all, in specialized translation, key terminology must be translated correctly and consistently. Dunne defines defective terminology as "terminology that is incorrect, inconsistent and/or ambiguous" (2007, p. 33). Translators and terminologists must avoid defective terminology by conducting proper terminology research before translating a text and by documenting that terminological research in terminology databases or termbases (TBs). Proper terminology management is a main factor in mitigating risks in the translation process that can arise due to defective terminology work. Thus, teaching translation-oriented terminology research and management must be integrated into the Translation classroom (CAT courses, PM courses, translation workshops) to train future translators and terminologists and provide them with the skillset that is necessary in the professional translation industry (Bilić, 2019). During the week when we focus on terminology management, students work on building termbases for their specialized final project source text with repetitive terminology that needs to be translated consistently. Students first extract key terminology from their final project source texts and then build termbases based on that text corpus. The course goal for the week is to learn proper terminology research and documentation as it relates to translation studies. Massion (2007) suggests the following basic steps to terminology management:

Here are four steps on the way to your own terminology: 1) define a terminology concept (organization and extent of the glossary, sources, tools, participants); 2) extract the terminology in the source language; 3) translate the terminology; and 4) distribute/manage the terminology. (p. 48)

According to Austermühl, "translation is a knowledge-based activity" and "terminological competence is translator competence" (2010, p. 3). A systemic concept-oriented approach to terminology management allows translators to gain knowledge in their area of specialization, and thereby achieve a higher level of competence in its specific terminology. In addition, terminology databases provide a "real-world context" and include context examples in both the SL and the TL. In contrast to bilingual dictionaries, translation-oriented 
terminology databases are corpus-based: the underlying terminology is extracted from relevant parallel texts and specialized parallel text corpora. Translator termbases are ideally corpus-based and concept-based. Students are required to structure their termbases using a concept-based approach, meaning that one term represents one concept. This is a means of quality management to avoid ambiguity and confusion that is caused by defective termbase entries that list different terms for the same concept or different concepts for the same term (Dunne, 2007, p. 33). The advantages of the concept-based approach to documenting terminology versus traditional dictionary entries are summarized in the table below:

Table 1: Dictionaries vs. concept-oriented termbases

\begin{tabular}{|l|l|}
\hline One term entry = many different concepts & One term entry = one concept \\
\begin{tabular}{|l|l} 
- Dictionaries and traditional glossaries list a term \\
in a given language and capture all its possible
\end{tabular} & $\begin{array}{l}\text { - A terminological entry in a termbase is based on the } \\
\text { concept to which the terms in various languages } \\
\text { meanings. }\end{array}$ \\
$\begin{array}{ll}\text { - This approach is not helpful when maintaining } \\
\text { specialized terminology where consistency is key. }\end{array}$ & $\begin{array}{l}\text { - Homonyms are listed in different terminological } \\
\text { entries. }\end{array}$ \\
& $\begin{array}{l}\text { - In addition, terminological entries contain detailed } \\
\text { information on terms, images, corpus-based context } \\
\text { examples, as well as "forbidden translations" for terms } \\
\text { in order to achieve terminological consistency. }\end{array}$ \\
\hline
\end{tabular}

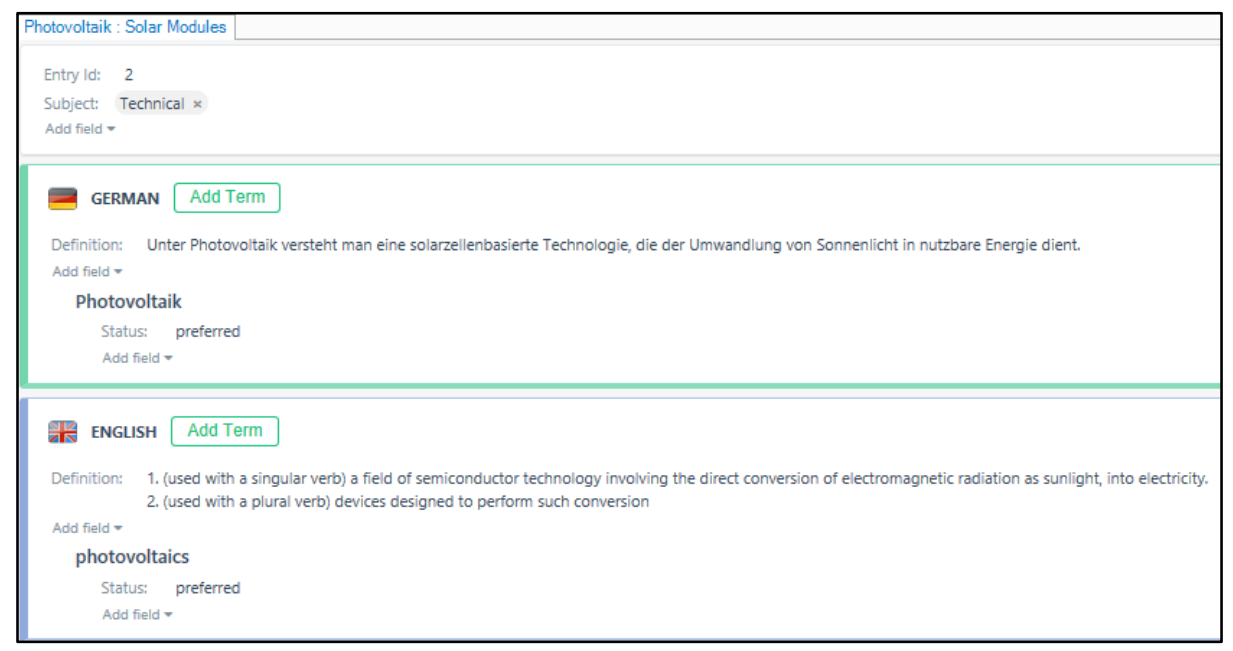

Figure 1: A German $<$ English term entry example - work in progress

Students create their own TB structure based on MultiTerm's bilingual glossary template, following the screencast tutorial and step-by-step homework instructions provided by the instructor. They are required to add several fields per source and target term in addition to the source and target term itself. Students first work in the MultiTerm software directly to set up the database and later add terms "on the fly" in the Studio translation editor. The entry fields that students add range from grammatical information, synonyms, context examples, definitions, and collocations to images and status information for specific key terms. The SDL MultiTerm termbases created during week 5 then provide the basis for students for translating their respective final project source texts throughout the semester. This way, students learn how to extract key terminology from their source text corpus and how to effectively research terminology and document key terms in a professional terminology management system. In line with the step-by-step approach to teaching translation technology, students are encouraged to keep adding fields to their existing term entries and enhance their termbases throughout the semester. 
Students have generally responded very well to this approach to terminology management and have created complex detailed concept-based terminology databases that have assisted them in translating their final projects.

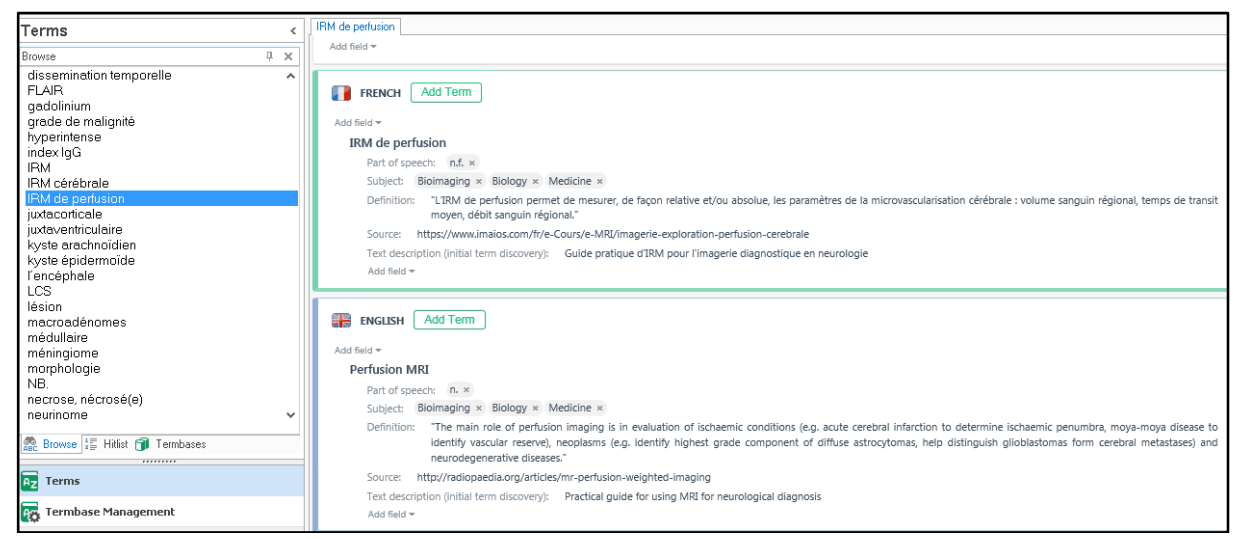

Figure 2: A French $<$ English term entry example - final submission

\subsection{Collaborative (online) working scenarios}

While there are in-house translators and translation company translators, many translators work as freelance translators, especially in the U.S. Freelance translators or so-called independent translators are self-employed, and "they either work for 'direct' clients (whom they invoice directly) or for agencies (or brokerage firms) that actually get the contracts and subcontract them to the freelancers" (Gouadec, 2007, p. 101). Translators assume various roles in the translation process. They work as project managers, terminologists, proofreaders, editors, reviewers, and as technical writers (Risku, 2006, p. 15). Risku further views translation as "Computer-Supported Cooperative Work", stressing that different independent actors take part in a translation project from start to finish with the "translation agency functioning as a network coordinator" (2006, p. 20). In such a collaborative translation lifecycle, project managers oversee the translation process and quality management is a crucial part of this collaborative process, especially because it involves so many different actors (e.g. clients, project managers, translators, terminologists, editors). In the modern translation industry, most of these actors in the translation process communicate entirely online. A possible work scenario includes project managers, terminologists, translators, and editors who all access the translation project which is stored on a server using the server version of CAT software. Several modern translation scholars have emphasized the importance of training future translators by using process-oriented rather than product-oriented teaching approaches. Kußmaul states that "we need methods for training translators" and that "these methods should be concerned with the actual process of translation" (1995, p. 4). Process-oriented approaches to translation are closely linked with functionalist approaches to translation that are based on Vermeer's Skopos theory (Reiß \& Vermeer, 1984). Kußmaul argues that by applying functionalist approaches to teaching translation and by encouraging students to not only analyse their final translation products but also the process that leads to them, students will internalize the steps in the translation process (1995, p. 150). My approach to teaching CAT tool technology has further been influenced by constructivist approaches to the teaching of translation, such as the method of cooperative translation. The method has been adapted for translation from the method of problem-based learning (PBL), a studentcentred, self-directed teaching method pioneered by Canada's McMaster University Medical School in the 1960s and further developed at medical schools throughout North America. In PBL, a small group (6-8) of medical students is presented with a complex, authentic learning task based on real-life 
scenarios (Barrows \& Tamblyn, 1980). Similarly, the cooperative translation technique moves away from the traditional and outdated model of the isolated translator toward a team-spirited, collaborative translation process. In cooperative translation, small groups of two to three students complete translation projects together as a group. Translation is seen as a decision-making and a problem-solving process that is best solved as a team. This method aims at teaching social competencies that represent an essential part of the modern translator's competency profile, where teamwork, accepting criticism and a willingness to compromise count alongside problem-solving skills. Translation teams fill out a translation protocol to indicate translation problems or issues faced during the process. In "student-centred" teaching, students take a more active and collaborative role in solving learning tasks. The instructor assumes the role of a tutor who allows students to work independently on solving translation problems. As a result, students learn communication skills and soft skills that are crucial in their professional lives (Kornelius et al., 2010). In the translation industry, teamwork represents a significant element of the modern translation process. Projects involve a close interaction with both colleagues and clients. Translation projects are oftentimes carried out by translators who work in teams and collaborate entirely online. In virtual collaborative translation classes, students share input by using modern Internet communication technology. Incorporating real-life working scenarios into the online computer-assisted translation classroom is another core component of the class. Toward the end of the term, students collaborate on workflow exercises that have been set-up by the instructor as the project manager on the memoQ server for the class. Students are paired as translator $<>$ editor teams and complete these workflow exercises by exchanging their translations via the memoQ server which is connected to all individual copies of memoQ on all student CAT class workstations. The project manager sets up Online Projects on the memoQ server. Translators and "reviewers" can then "check out" these projects from the server directly. Possible other workflows may include students assuming the roles of project managers, terminologists, translators, and proofreaders/editors.

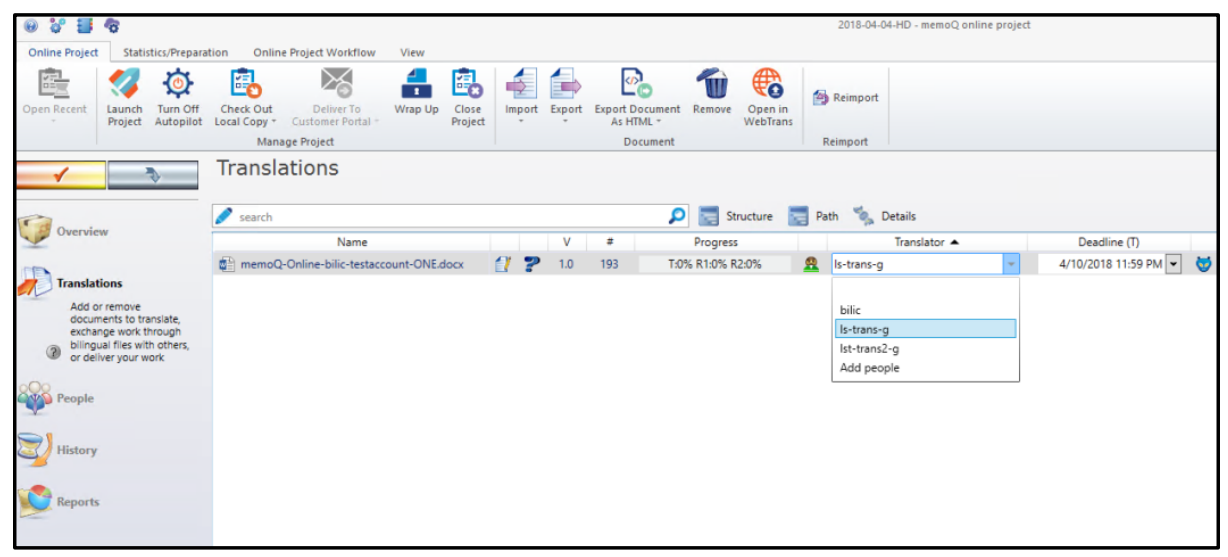

Figure 3: Setting up an online project on the memoQ server

In a standard memoQ server workflow scenario there are three persons involved: The instructor sets up a translation project for each student. All student translators then create a termbase for their project, complete the target text, and send the final product (comprising the translated text, the TM, and the TB) to their assigned student editors who work in the same language pair. 


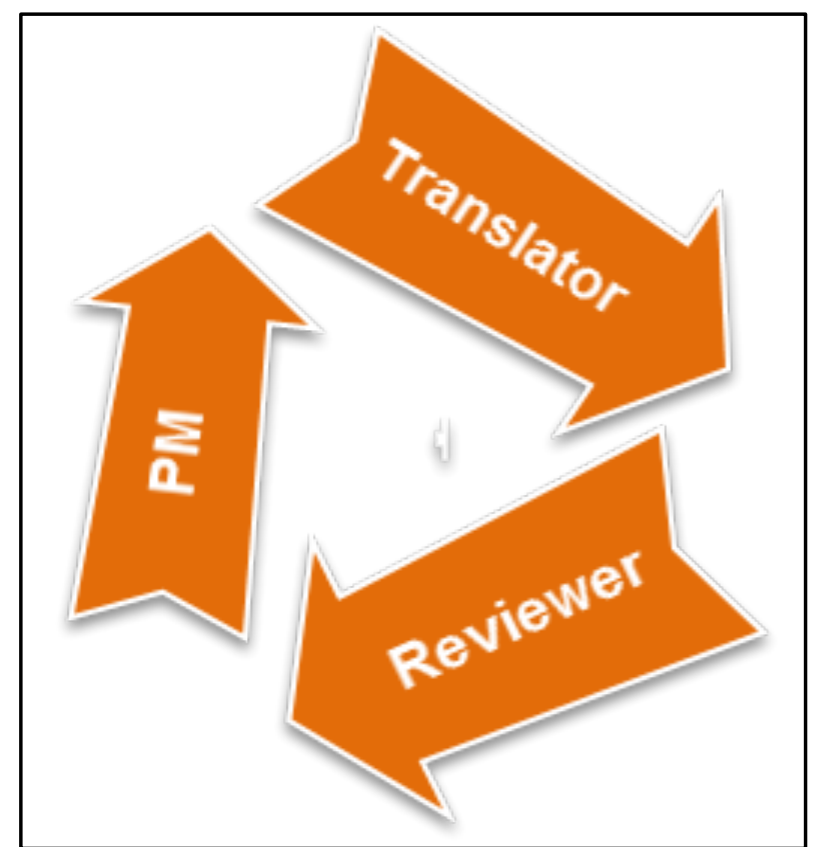

Figure 4: Translator/Reviewer workflow project using memoQ server

The students who perform the reviewer role carefully edit their classmates' target text, and then have the option to forward the edited translation to the project manager immediately or return it to the student translator with suggestions and edits.

8. Now "Deliver/Return" the reviewed translation to the PM/server just like you submitted your translation: Click on "Deliver/Return" in the Documents tab or right-click your Word file and choose "Deliver/Return."

9. You will receive the message below:

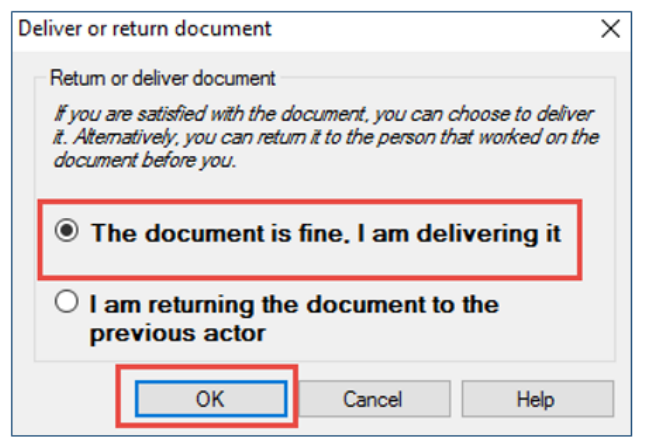

Figure 5: Homework instruction example (steps 8 and 9) for a memoQ server workflow exercise

Workflow exercises using server-based software such as memoQ server give students the opportunity to assume different roles in the translation process. Students are collaborating entirely online, using the memoQ server software that is hosted on a dedicated university server. All students are involved in both their own and their partner's server-based translation lifecycle from start to delivery. Students gain experience translating and reviewing translation projects that are entirely server-based. They also gain experience communicating and exchanging online projects with their assigned partner, replicating a real-life translation lifecycle using modern technology. 
11. Your reviewed translation will now be delivered to the server and the PM (me). The workflow status will change from "Workflow status: review 2 (in progress)" indicated by a red and yellow pencil to "Workflow status: completed" indicated by a green check mark.

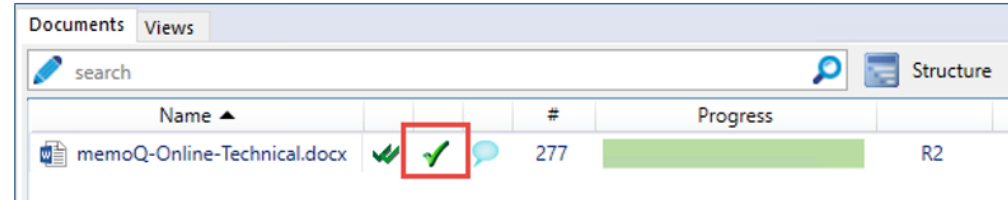

12. A completed project has a double check mark on the server. This is what it looks like for the PM who can track progress anytime:

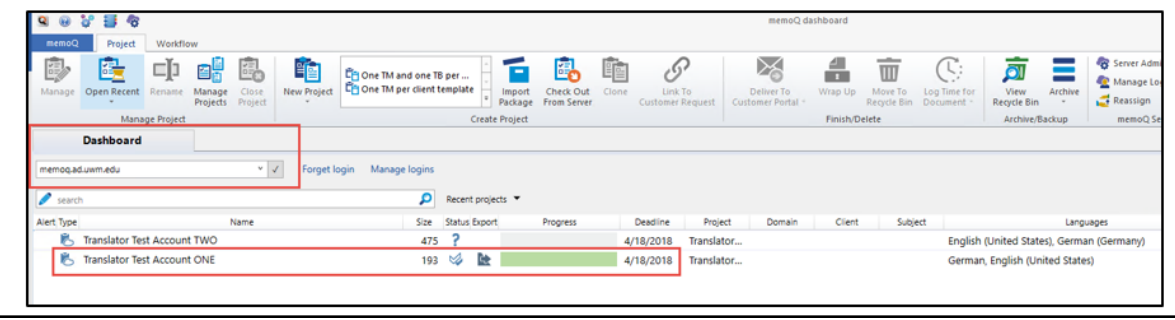

Figure 6: Homework instruction example (steps 11 and 12) for a memoQ server workflow exercise

\section{4 (Online) student feedback}

The student success rate in the Online CAT Classroom is high: $90 \%$ of all students enrolled in the Online CAT courses in the years between 2016 and 2019 passed the SDL Level 1 Certification which is an integrated part of the class. Students have access to the virtual lab server and CAT software for three months following the class and have the opportunity to complete further SDL and memoQ certifications. At the end of the class, students submit an anonymous course evaluation questionnaire. This input from the students is crucial for potentially revising the online class regarding its course design, structure, and contents. The responses are summarised graphically in the figure below.

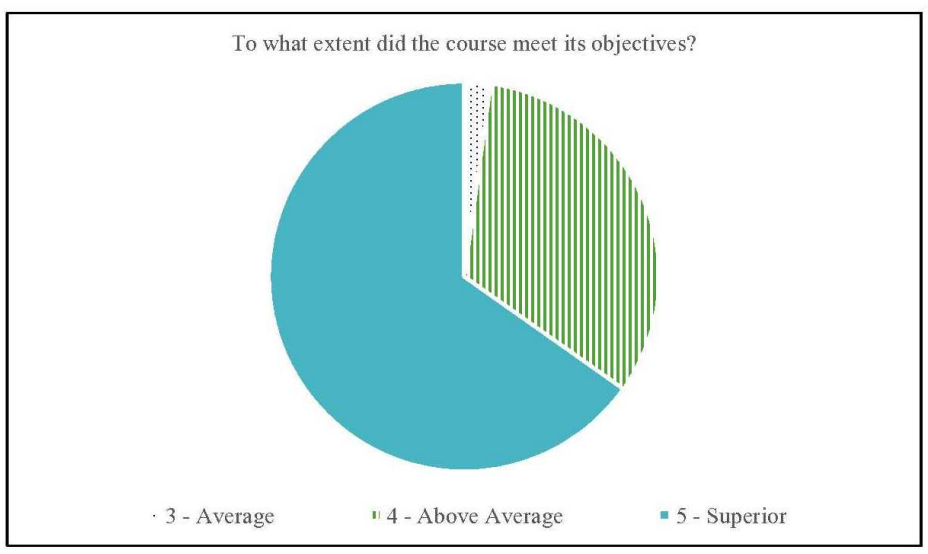

Figure 7: Overall course satisfaction - student evaluations

In all, 57 students have taken the Online Computer-Assisted Translation class over the years between 2016 and 2019. Of that number, 46 (about 80\%) have submitted the course evaluation via an anonymous online survey application. Overall, 30 (approx. 65\%) of the responding students gave the course a "superior" rating, while 15 (approx. 33\%) thought it was "above average" (UWM, 2019).

Students noted the benefits of working with a dedicated virtual lab server. Among the comments compiled in the UWM Qualtrics Course Evaluations 2016-2019, one wrote that "I have never had the opportunity to access a virtual computer lab before and I couldn't believe how well it worked. I actually didn't 
know that that was even possible. It really makes technology accessible for students." (UWM, 2019). The graduate students also noted that the hands-on software exercises were among the most beneficial parts of the course. For example, one remarked that

what I enjoyed most about this course was the ability to interact with important technology that's being used in our field today. I now feel more prepared to enter the job market because I'm familiar with these tools and have a strong foundation on which to build. (UWM, 2019)

Another student pointed out that "I walk away from this class with many new tangible skills that I can apply in real life" (UWM, 2019). Students also mentioned that course communication and online collaboration was smooth and that troubleshooting software issues online via Skype tutoring, screen sharing, and through communication via the online discussion boards helped prepare them for tackling such software errors in the future. It needs to be pointed out that the limitations to this study are that the teaching methods are evaluated by the student success rate and the student questionnaires. Ninety percent of students taking the class in the years between 2016 and 2019 passed the SDL Certification Level 1 , and the student evaluations were generally very positive. However, the study results are based on a relatively small group of 57 graduate students of Translation and cannot be generalized to include a wide population of online students.

\section{Conclusion}

Teaching translation technology online holds many challenges as it includes troubleshooting software errors entirely online. The virtual CAT classroom can only function well when both students and the instructor are actively communicating via the course platform, online meeting rooms, Skype, or communication channels within the CAT server software. Students need to be proactive and raise software-related questions and make the instructor aware of potential technological issues before the homework due date. The instructor must respond to student questions in a timely manner and offer at least twice as many Skype office hours as for a regular (online) class. In order to teach translation technology in the virtual classroom successfully, CAT exercises must be tested on the students' remote workstations in advance and the course content must be developed in its entirety before course launch to ensure a positive learning experience for students. The teaching methods and effective course design can be summarized in accordance with Table 2 below.

Table 2: Keys to a successful student/teacher experience

\begin{tabular}{|c|c|c|c|}
\hline $\begin{array}{l}\text { Organization } \\
\text { course materials }\end{array}$ & $\begin{array}{l}\text { Instructional design } \\
\text { and } \\
\text { strategies }\end{array}$ & $\begin{array}{l}\text { Learning activities } \\
\text { and homework } \\
\text { assignments }\end{array}$ & $\begin{array}{l}\text { Effective use } \\
\text { online setting }\end{array}$ \\
\hline $\begin{array}{l}\text { - Well-organized, clear } \\
\text { and complete course } \\
\text { materials } \\
\text { - Detailed syllabus and } \\
\text { course instructions }\end{array}$ & $\begin{array}{l}\text { - Instructor establishes a } \\
\text { strong presence on the } \\
\text { course platform. } \\
\text { - Instructor encourages } \\
\text { students to participate } \\
\text { actively in the online } \\
\text { discussions. } \\
\text { - Instructor provides } \\
\text { feedback on a weekly } \\
\text { basis. }\end{array}$ & $\begin{array}{l}\text { - Students work on } \\
\text { translations and } \\
\text { terminology databases } \\
\text { using CAT software } \\
\text { - Homework assignments } \\
\text { include "client } \\
\text { instructions" }\end{array}$ & $\begin{array}{l}\text { - Foster a collaborative } \\
\text { environment } \\
\text { (through } \\
\text { lectures, discussion, } \\
\text { homework, etc.) } \\
\text { - Creating an online } \\
\text { community } \\
\text { - Group work: Student } \\
\text { translation teams } \\
\text { collaborate entirely online } \\
\text { using modern technology. }\end{array}$ \\
\hline
\end{tabular}

Teaching translation technology in the virtual classroom is often challenging, but it also has many benefits as it mimics the real-life working 
scenario of most professional translators and prepares students for the demands of the translation industry. Many students have no or little experience using CAT tools, even if they have worked as freelance translators. The course offers a step-by-step approach to using CAT tools, and students can work on exercises at their own pace and depending on their comfort level with using technology. In the Online Computer-Assisted Translation Classroom students learn how to use CAT software effectively as professional translators by creating translation memories and terminology databases for specialized texts to ensure that consistent terminology is used throughout a target text. In addition, students also experience the translation process from the point of view of project managers and editors who prepare the source text material for translation using a CAT tool as a first and important step of quality management in translation. Students also edit translations of their classmates and exchange comments on one another's translation work using the server edition of memoQ. Thus, students learn about CAT tools in an ideal work scenario, where translators and proofreaders/editors work together and give each other feedback. In the online CAT classroom, students have the opportunity to explore all of these three phases by completing all steps in the translation workflow using the serverbased version of memoQ for example, and by effectively communicating with the instructor and their classmates in the virtual classroom.

\section{References}

Austermühl, F. (2010). A collaborative approach to the teaching of terminology management. T21N Translation in Transition, 2010-09, 1-22. Retrieved from http://www.t21n.com/homepage/articles/T21N-2010-09 Austermuehl.pdf

Barrows, H. S., \& Tamblyn, R. M. (1980). Problem-based learning: An approach to medical education. New York: Springer Pub. Co.

Bilić, V. (2015, July). Teaching terminology and computer-assisted translation online. Paper presented at the XXth European Symposium on Languages for Special Purposes, Vienna.

Bilić, V. (2016-2019). Translation 726. Course syllabus and course materials. University of Wisconsin-Milwaukee.

Bilić, V. (2017, April). Teaching translation technology in the virtual classroom. Paper presented at the Monterey Forum 2017, Monterey.

Bilić, V. (2019, May). Building terminology databases: web-based, corpus-based or concept-based? Paper presented at the Monterey Forum 2019, Monterey.

Bowker, L. (2014). Computer-aided translation. Translator training. In S. Chan (Ed.), Routledge encyclopedia of translation technology (pp. 88-104). New York: Routledge.

Chan, S. (Ed.). (2018). The human factor in machine translation. New York: Routledge.

Childress, M. (2007). Terminology work saves more than it costs, MultiLingual, 18(3) (April/May), 43-46.

Dunne, K. (2007). Terminology: ignore it at your peril. MultiLingual, 18(3) (April/May), 32-38.

Göpferich, S., \& Jääskeläinen, R. (2009). Process research into the development of translation competence: Where are we, and where do we need to go? Across Languages and Cultures, 10(2), 169-191. Retrieved from https://akademiai.com/ doi/abs/10.1556/Acr.10.2009.2.1

Gouadec, D. (2007). Translation as a profession. Amsterdam/Philadelphia: John Benjamins.

Gracia García, R. (2005). Teaching interpreting online. In M. L. Romana García (Ed.), II AIETI. Actas del II Congreso Internacional de la Asociación Ibérica de Estudios de Traducción e Interpretación (pp. 438-449). Madrid: AIETI, Retrieved from http://www.aieti.eu/wp-content/uploads/AIETI_2_RAGG_Teaching.pdf

Keller, N. (2014). Translation-Memory-Systeme 2014 unter der Lupe. Mitteilungen für Dolmetscher und Übersetzer, 60(2), 8-116.

Kornacki, M. (2018). Computer-assisted translation (CAT) tools in the translator training process. Berlin: Peter Lang. 
Kornelius, J., Orbán, W., \& Stewart, J. (2010). Cooperative translation in the paradigm of problem-based learning. T21N Translation in Transition, 2010-01, 1-29. Retrieved from http:/www.t21n.com/homepage/articles/ T21N-2010-01-Stewart, Orban,Kornelius.pdf

Krüger, R. (2016). Contextualising computer-assisted translation tools and modelling their usability. trans-kom, 9, 114-148. Retrieved from http://www.trans-kom.eu/ bd09nr01/trans-kom_0901_08 Krueger_CAT.20160705.pdf

Kußmaul, P. (1995). Training the translator. Amsterdam: John Benjamins.

Larson, D. K., \& Chung-Hsien S. (2009). Comparing student performance: online versus blended versus face-to-face. Journal of Asynchronous Learning Networks, 13(1), 31-42. Retrieved from https://files.eric.ed.gov/ fulltext/EJ837556.pdf

Leone, L. (2015). The online literary translation workshop. Translation Review, 92(1), 86-98. Retrieved from https:/www.tandfonline.com/doi/ full/10.1080/07374836. 2015.1094434

Lim, D. H., Morris, M. L., \& Kupritz, V. W. (2007). Online vs. blended learning: differences in instructional outcomes and learner satisfaction. Journal of Asynchronous Learning Networks, 11(2), 27-42. Retrieved from https://files. eric.ed.gov/fulltext/EJ842695.pdf

Lowe, E. (2015, July). Online course design and development \& teaching methods and ethics of translation online. Paper presented at the XXth European Symposium on Languages for Special Purposes, Vienna.

Massion, F. (2007). Terminology management: a luxury or a necessity? MultiLingual, 18(3), 47-50.

McCallum, M., \& Puchner, S. (2017). Transitioning from student to translator. ATA Chronicle, XLVI(2) (Mar/Apr), 24-26. Retrieved from http:/www.atanet.org/ chronicle-online/featured/transitioning-from-student-to-translator/

McKay, C. (2006). How to succeed as a freelance translator. Morrisville, NC: Lulu.

Mellinger, C. (2014). Computer-Assisted Translation: An Empirical Investigation of Cognitiv Effort (Doctoral dissertation). Retrieved from https://etd.ohiolink.edu/ pg_10?::NO:10:P10_ETD_SUBID:94337

Parker, R. E. (2013). Rédesigning courses for online delivery: design, interaction, media \& evaluation. Bingley, UK: Emerald Publishing.

Reiß, K. \& Vermeer, H. J. (1984). Grundlegung einer allgemeinen Translationstheorie. Tübingen: Niemeyer.

Risku, H. (2006). Risku, H. (2006). Cooperation and quality assurance in technical translation projects. Language at Work - Bridging Theory and Practice, Vol. 1(1), 1-10. https://doi.org/10.7146/law.v1i1.6234

Sauberer, G. (2009). Terminology management in translation. Workshop presented at TermNet International Network for Terminology conference, New Dehli. Retrieved from https:/www.termnet.org/english/events/ itaindia_workshop.php

Starlander, M. \& Morado Vázquez, L. (2013). Training translation students to evaluate CAT tools using Eagles: a case study. In Aslib: Translating and the Computer 35, 1-10. Retrieved from https://archive-ouverte.unige.ch/unige:35622

Sun, S. Y. H. (2014). Learner perspectives on fully online language learning. Distance Education, 35:1, 18-42. Retrieved from https:/www. tandfonline.com/doi/abs/10. 1080/ 01587919.2014.891428

Sun, S. Y. H. (2016). Learntime and learning place-focused forward-oriented design for learning in technology-enhanced classrooms. Distance Education, 37:3, 349-365. Retrieved from https://www.tandfonline.com/ doi/abs/10.1080/01587919. 2016. 1233051

Terando, L. (2014, November). Quality control for online course development/teaching. Teaching Translation Online: Quality Assessment and Control. Paper presented at the $55^{\text {th }}$ ATA Conference. Chicago.

UWM (University of Wisconsin-Milwaukee). (2019). Qualtrics Course Evaluations (2016-2019). Student course evaluations for Translation 726 taught by V. Bilić. Unpublished. Accessible on internal UWM platform.

\section{Image credits:}

Tables 1 and 2: Bilić, V., 2019.

Figures 1, 3, 4, 5, 6, 7: Bilić, V., 2016-2019.

Figure 2: Breckenridge, C., 2016. 